\title{
Conformorality
}

\section{A Study on Group Conditioning of Normative Judgment}

\begin{abstract}
How does other people's opinion affect judgments of norm transgressions? In our study, we used a modification of the famous Asch paradigm $(1951,1955)$ to examine conformity in the moral domain. The question we addressed was how peer group opinion alters normative judgments of scenarios involving violations of moral, social, and decency norms. The results indicate that even moral norms are subject to conformity, especially in situations with a high degree of social presence. Interestingly, the degree of conformity can distinguish between different types of norms.
\end{abstract}

Keywords: normative judgments, moral norms, conformity, nonverbal communication

\section{Introduction}

What is worse? Stealing from your neighbor, tipping in Japan or spitting in your glass before drinking? Most people will have no hesitation in answering this question. Perhaps, they may also explain that those behaviors involve different kinds of norms. The first situation seems to concern a moral norm, which holds in all cultures and whose normative force does not depend on people's expectations and preferences. The second involves a social norm, which holds only in particular contexts and whose normative force depends on people's expectations and preferences. The third example, similarly to the first one, involves a type of behavior that is likely to elicit a wave of disgust independently of context or people's preferences and expectations, but just like a social norm, it involves a matter of relatively low seriousness.

This intuitive taxonomy roughly corresponds to a distinction between different kinds of norms, which emerges from the literature on normative judgment in moral psychology (e.g. Bicchieri, 2006; Elster, 2009; Haidt, Koller, and Dias, 1993; Nichols, 2002; Turiel, 1977). Although 
there are differences in the way particular researchers individuate different kinds of norms, many would agree that there are features that distinguish moral, social and what can be called "decency norms.” For example, Turiel (1983, 2002) and his collaborators (Nucci, 2001; Smetana, 1993) proposed that people neatly distinguish between moral and conventional norms along four main features: (in)dependence on authority, scope, seriousness of violation and grounds for justification. According to this distinction, violations of moral norms would be judged as wrong independently of the pronouncements of authorities; moral norms would have universal scope, treated as holding in all places and at all times; violations of moral norms would be judged as seriously bad; and justification of such norms would refer to the harm or injustice suffered by the victim when they are violated. Conventional norms, by contrast, would be considered to be authority-dependent, limited in scope, their violations would be less serious than moral violations and their justification would tend to involve considerations such as the maintenance of social order rather than the harm or injustice suffered by some victim.

Numerous studies have demonstrated that the distinction between moral and conventional norms emerges early in human psychology, around three and a half year, and is present across different cultures (e.g. Turiel, 1983, 2002; Smetana, 1993; Nucci, 2001). The conclusion that is often drawn in the literature is that moral norms and conventional norms, as characterised by Turiel and collaborators, form different kinds of norms, which can be neatly distinguished by human moral psychology (see Nado et al., 2009, for a critical discussion).

Recent research has disputed this conclusion, indicating that the features that allow us to distinguish between different kinds of norms can be more subtle and intricate than what Turiel and colleagues suggested. Kelly et al. (2007), for example, showed that when experimental participants are asked to evaluate the violations of moral norms in cultures and societies far away in both time and space, they often judge them to be tolerable. Moreover, Nichols (2002) and Haidt (2001) showed that disgusting behaviors may be perceived as seriously bad as moral transgressions, albeit they do not involve harm or injustice to others. Such disgusting behaviors might be governed by an 
idiosyncratic kind of emotionally-laden norms, distinct from moral and social norms (Nichols, 2004), which we call “decency norms."

Furthermore, judgments about certain types of normative behaviors, but not about others, may well be more resistant to group pressure. Intuitively, given that moral norms are typically assumed to be non-negotiable, we might expect that your judgment about, for example, stealing will be less easily affected by conformity, compared to a judgment about a social norm such as tipping or about a decency norm such as spitting in your glass before drinking. To our knowledge, it has never been experimentally investigated whether different kinds of norms can be distinguished by the degree to which they are affected by peer-group judgment. Answering this question will contribute to make progress both in understanding which features allow our mind to selectively distinguish between different kinds of norms, and specifically how social cues impact normative judgment.

In light of previous evidence about the developmental and cultural robustness of moral norms, we hypothesize that the norms that are most resistant to peer-group judgment will be moral norms - as characterised by Turiel and collaborators. Norms that are the least resistant to peer-group judgment will be social norms - corresponding to Turiel's conventional norms. With respect to decency norms, if they are found to be significantly different from moral norms in their resistance to conformity effects, then, on the one hand, disgust might not be essential to moral judgment, and, on the other hand, disgust would probably be insufficient to lead people to morally disapprove of a behavior where no harm or injustice are involved.

To test these hypotheses, the present study employed, for the first time in moral psychology, Asch's $(1951,1955)$ group conditioning paradigm. We compared participants' individual judgments concerning the violation of moral, social, and decency norms, to the judgments the same participants gave in the presence of other people expressing different opinions.

Finally, given that nonverbal, social cues such as eye contact, facial expressions and tone of voice seem to play a crucial role in defining in-group social identity and its prototypical (normative) 
behavior (Hogg and Reid, 2006) as well as in facilitating reaching agreement within a group (Hiltz, Johnson and Turoff, 1986), we hypothesised that the degree of awareness of the other persons-socalled social presence (Short et al., 1976) — in the group conditioning situation might have an effect on conformity. To identify the possible effects of available nonverbal display, we tested whether being unable to see and hear each other results in a lower degree of conformity.

\section{Test of Material}

The test of the experimental material consisted in evaluating 30 scenarios that described a transgression of some norm. The scenarios were based on examples that are found in the philosophical and psychological literature. They included descriptions of behavior involving, for example, some injustice or harm to other people (for what we pre-experimentally took to be moral norms), the infringement of a mutual agreement concerning fairness, reciprocity or equity (for what we took to be social norms), and behaviors associated with physical uncleanliness, "creepy crawlies" or non-standard sexual practices (for decency norms). The aim was to test if the scenarios would be interpreted by the subjects as instances of moral, social, and decency norms, respectively.

In the test, we also considered the potential impact of personal distance to the perpetrator/victim of the norm transgressions. One could argue that violations that personally involve the participant could trigger emotional processes (Greene et al., 2001, 2004) that might be difficult to evoke with a scenario-based experimental method. If that is the case, we might expect respondents to evaluate differently scenarios concerning strangers (typically employed in moral psychology) to those where the victim and the perpetrator are known to the respondent.

\subsection{Method}

Participants. Sixty-eight Dutch students (57 female) were recruited from the undergraduate student population at the Tilburg University. They were randomly divided between two conditions and received course credits for their participation. 
Design and Instrumentation. The test of the material had a 2 x 3 mixed design with Distance (scenario concerned a stranger as opposed to a friend/family member) as the between-subject independent variable and Norm Type (moral, social, decency) as the within-subject independent variable. The 30 scenarios were presented in English and described violations of moral, social and decency norms (10 scenarios per Norm Type, see Table 2 for examples and Table 3 for a list of the transgressions employed, classified per type of norms with references to the literature they came from or were based on). The participants were asked to evaluate the scenarios with respect to the following four items, each operationalized in terms of a 7-point scale anchored at the ends with (1) strongly disagree and (7) strongly agree: Badness ("X's behavior is very bad"), Disgust ("What X did is nauseating"), Time/Place ("In a different time/place, it is OK to do what X did") and Authority ("If the law allows it, it is OK to do what $\mathrm{X}$ did"). These items were based on the properties identified by Turiel (1977), Kelly (2007) and Nichols (2002) as characteristic features of different types of norms. In the Stranger condition, the scenarios concerned unknown individuals with invented names; in the Friend/Family condition, the names were replaced with phrases such as "your roommate," "your best friend" or "your parents."

Procedure. The test was administered online and presented as a study of Dutch taboo subjects. The participants were invited to read each scenario as if it were describing a situation that actually happened.

\subsection{Results}

We analyzed the results with mixed ANOVAs with Norm Type and Distance as independent variables and the score on each of the four items as the dependent variable. The data showed no significant main effects of Distance for Badness, $F(1,66)=2.945, p=.091$, for Disgust, $F(1,66)<$ $1, p=.579$, for Time/Place, $F(1,66)<1, p=.620$, and for Authority, $F(1,66)<1, p=.521$. There were also no significant interaction effects between the variables Norm Type and Distance for Disgust, $F(2,132)<1, p=.430$, for Time/Place, $F(2,132)=2.850, p=.061$, and for Authority, 
$F(2,132)=1.959, p=.145$. There was an interaction effect between Norm Type and Distance for Badness, $\mathrm{F}(2,132)=4.527, p=.013$, however, the effect was small $\left(\eta_{p}^{2}=.064\right)$.

These results indicate that scenarios that involved the participants' friends and family members were not judged differently than the scenarios involving strangers. The scales evaluated for each scenario distinguished between three Norm Types as summarized in Table 1. For the property Badness, Decency and Time/Place, the three types of norms differed significantly from each other: for Authority, a pairwise comparison showed a difference between moral and social violations, and decency and social violations, but no significant difference between moral and decency violations. Finally, we inspected the correlations between the scores assigned to scenarios within a Norm Type, using Badness for moral norms, Disgust for decency norms and Time/Place and Authority for social norms. The analysis showed no outliers within the categories (scenarios that would be negatively correlated with other scenarios in the category with respect to the distinguishing property). The Cronbach's alpha coefficients were $\alpha=.617$ for moral violations on the Badness-scale, $\alpha=.814$ for decency violations on the Disgust-scale, and $\alpha=.716$ on the Time/Place-scale and $\alpha=.745$ on the Authority-scale evaluated for the social norm violations.

\subsection{Discussion}

The results of the material test show that characteristic properties of three types of norm violations, which have been identified in the literature (the seriousness of the violation, its dependence on time/place and on an authority and the feeling of disgust it evokes) distinguish between scenariotypes employed in the test and thus validate our intuitive original classification of the scenarios, which was based on the literature. The participants were not more sensitive to scenarios involving a familiar person compared to those concerning a stranger and the distinction was not taken into consideration in the subsequent experiment, in which we employed the thirty scenarios from the material test. 


\section{Experiment}

\subsection{Method}

Participants. Ninety-seven Dutch native speakers (66 female), all with a good command of English, between the ages of 19 and 49, were recruited from the undergraduate student population at the Tilburg University and received course credit for their participation.

Design and Instrumentation. The experiment had a mixed 3 x 3 design, with Norm Type (moral, social, decency) as the within-subject variable and Social Presence (high, low and control) as the between-subject variable. The questionnaire consisted of the 30 short scenarios described above and 10 distractors. The distractor items had content similar to the experimental items in that they involved different kinds of norm violations.

The participant's judgment was measured on a 7-point scale anchored at the ends with (1) "strongly disapprove" and (7) "strongly approve," with participants indicating their acceptability judgment for each scenario, first in an individually completed online questionnaire and, two weeks later, in a group condition with three confederates. In the online version of the questionnaire, participants were also asked to indicate for each scenario if they were certain of their judgment (yes/no).

For the 30 experimental items, the confederates' answers employed in the group condition were chosen using the mean of the participants' answers in the first measurement, with two scale points added to the mean (in the direction opposite to the conventional answer). The confederate answers were unanimous on the 30 experimental items and differed for the 10 distractor items. In the control condition, participants merely filled out the online questionnaire twice with a two-week period in between. For the first measurement in the individual condition, we used two sequences of the online questionnaire to test for possible order effects. In the second sequence, the questions were presented in a reverse order.

Procedure. In the group condition with high social presence, the participants were seated together with three confederates and they could see each other's expressions and hear each other's 
voice. In total, 24 students, both male and female, acted as confederates. The experimental leader (a female for half of the trials and a male for the other half) read each scenario and the participants gave their answer in the order: confederate 1 - confederate 2 - participant - confederate 3 . The participants were informed that the answers they gave online were lost due to a server error and had to be collected again. In order to avoid differences in cognitive load between the first and the second measurement, the participants were supplied with the text of the scenarios on paper.

In the condition with low social presence, the participants were seated in front of a computer screen in the same room as the confederates but could not see their face. In order to exclude vocal cues, they all indicated their judgments for each scenario by selecting their answer on the screen, where both the scenarios and the answers of the confederates were presented. At the end of each session, the participants were interviewed and debriefed. None of the participants reported having difficulties in judging the scenarios.

\subsection{Results}

A Wilcoxon-Mann-Whitney test of judgments per scenario collected during the first measurement revealed no effect of presentation order on participants' judgments. The data from the first measurement in all three conditions, summarized as the mean value of the participants' judgments per scenario, were used to examine the homogeneity of variance for the three types of norms. The Levene Statistic showed that the assumption of equal variances was valid, indicating no systematic differences in answer distributions.

In order to test if all three types of scenarios were judged with the same certainty, we first compared the categorical data indicating participants' certainty of their approval judgments. There was no significant difference between the three scenario types, $\chi^{2}(2)=.16, p=.920$; for most scenarios $(92.7 \%)$, the participants indicated to be certain of their judgment.

In the subsequent analyses comparing the first and the second measurement, we excluded cases where the participant had the same judgment during the first measurement as the confederates 
in the group condition (13\% of the total of 2910 experimental trials, distributed equally over the three Norm Types).

We calculated Conformity (C) using the approval judgments given by the participants in the individual $\left(\mathrm{M}_{1}\right)$ and the group condition $\left(\mathrm{M}_{2}\right)$ and the confederates' opinion $(\mathrm{O})$, as $\mathrm{C}=\left|\mathrm{O}-\mathrm{M}_{1}\right|-\mid$ $\mathrm{O}-\mathrm{M}_{2}$. A positive value of $\mathrm{C}$ represents instances where the participant's judgment shifted closer to the confederates' opinion, a negative number stands for cases where the distance increased and 0 for cases where the distance remained the same.

Given that the dependent variable Conformity was not normally distributed (Shapiro Wilk's tests $<.05$ ), we used nonparametric tests throughout. We first examined whether male and female participants differed in their overall Conformity scores in the two conditions involving confederates. A Mann-Whitney $U$ showed no significant effect for gender $(U=374.00, z=-1.43, p$ $=.154)$. We used the Kruskal Wallis test to analyze the difference between the experimental conditions with high and low social presence and the control condition. A Mann-Whitney test with Bonferroni correction showed that the condition with high social presence differed from the Control condition for all three Norm Types, as well as the Total Conformity. The condition with low social presence differed from the Control condition in the case of Moral and Decency Conformity, but not for Social Conformity and the Total Conformity (see Table 4).

In order to examine the difference between the three Norm Types (moral, social, and decency) in detail, we used the Friedman test to compare the level of Conformity separately in the two experimental conditions, with high and low social presence. The analysis showed that the three Norm Types differed only in the condition with high social presence $\left(\chi^{2}(2)=7.09, \mathrm{p}<.05\right)$, but not in the condition with low social presence $\left(\chi^{2}(2)=2.97, p=.227\right)$-see Table 5 . In the condition with high social presence, participants conformed the most to the scenarios describing social violations $(\mathrm{Mdn}=.600)$, compared to decency violations $(\mathrm{Mdn}=.546)$ and moral violations $(\mathrm{Mdn}=.400)$. Wilcoxon tests with the Bonferroni correction (effects reported at a .0167 level of significance) showed that Conformity to judgments of moral violations differed from Conformity to social $(\mathrm{p}=$. 
003, $\mathrm{r}=-.471)$ and decency violations $(\mathrm{p}=.008, \mathrm{r}=-.417)$, but Conformity to judgments of social violations did not significantly differ from Conformity to decency violations $(\mathrm{p}=.187, \mathrm{r}=-.160)$. Finally, we ran a secondary analysis of the consistency of answers across measurements, calculated as the absolute difference between the participant's first and second measurement (independently of the confederates' answers). The results showed that similarly to the Conformity measure, the stability of answers was higher for moral scenarios compared to the other two types (see Table 6).

\section{General Discussion}

Earlier research in psychology has examined, on the one hand, the effects of authority on obedience and norm compliance (Milgram, 1963), in-group/out-group effects on moral behavior (Tajfel et al., 1971), and the consequences of emotional cues on people's normative judgments (Schnall et al., 2008; Wheatley and Haidt, 2005). On the other hand, research studies on humans and nonhuman primates have shown that both species tend to adjust their behavior and beliefs toward others in their social circles (Cialdini and Goldstein, 2004; Whiten, Horner and de Waal, 2005). In humans, conformity can affect judgments ranging from perceptual line-length estimates (Asch, 1951) to more complex behaviors, such as energy saving (Schultz, Nolan, Cialdini, Goldstein and Griskevicius, 2007).

Combining both threads of research on normative judgment and conformity effects in an original way, our experiment focused on understanding the effects of peer pressure on individuals' normative judgments. The results of our experiment indicate that while all normative judgments tend to be affected by peer-group judgment to some degree, the effect is the strongest for social and decency norms, which are most likely to be influenced by peer-group conditioning. Moreover, the effect is especially pronounced in situations involving a higher degree of awareness of others, operationalized in terms of the availability of nonverbal display. The degree of conformity to other people's normative judgment as such can then be used to independently motivate the distinction between moral norms and social norms proposed by Turiel and collaborators. 
Our findings are coherent with previous research both on conformity effects in computermediated communication (Smilowitz et al., 1988; Bordia, 1997; Cinnirella and Green 2007; Laporte et al., 2010), as well as with studies conducted by Bicchieri (2008) and Cialdini et al. (1991) on the effects of expectations about other people's compliance with a norm.

To explain our main results, it can be suggested that the predisposition we have towards conformism to common behaviors and shared opinions of our own group is counterbalanced by the robust influence that a specific kind of norms, that is moral norms, has on our mind. Accordingly, although cultural-evolutionary models suggest that conformity has adaptive value under a range of conditions (Henrich and Boyd, 1998), behavior and opinions that involve violations of moral norms are more insulated from conformity effects. For by conforming to behaviors and shared opinions that involve violations of moral norms, members of a group would not increase survival likelihood at both the personal and the group level. Hence, the degree of dependency on other people's judgments makes it possible to reliably distinguish moral norms from different types of norms. On this basis, it can be suggested that moral norms constitute a natural kind in human moral psychology.

Furthermore, the fact that decency norms appear to be less stable than moral norms lends support to critical reviews according to which there is weak evidence that disgust is a moralizing emotion (Huebner et al., 2009). Although disgust may be implicated in moral judgment, it is probably neither sufficient nor necessary for moralization to occur (Royzman et al., 2009).

A number of variables, including group size, group composition in terms of gender and age, as well as cultural background of the participants may influence the outcome of group conditioning experiments and should be explored in future studies of conformity to judgments of norms. However, if human psychology is selectively sensitive to recognize and implement moral norms, which might constitute a cognitive domain robust to conformity effects, then our main result should be found across different groups and cultures. 
The language of the experiment might also be a factor; in our study, we presented English scenarios to Dutch participants. Even though their knowledge of English was good, the fact that they were evaluating norm transgressions in a non-native language may have reduced the impact of our manipulation (Puntoni, de Langhe and Van Osselaer, 2008). Arguably, this might affect decency norms more than moral ones.

Additional research is also needed to validate the scenario-based technique employed here by linking it to behavioral data collected in natural and simulated (game) settings (Van Lankveld et al., 2011), possibly using methodology that has been previously employed to determine personality profiles.

\section{References}

Asch, S. (1951). "Effects of group pressure on the modification and distortion of judgments." In H. Guetzkow, ed., Groups, leadership and men (pp. 177-190). Pittsburgh, PA: Carnegie Press.

Asch, S. (1955). “Opinions and social pressure.” Scientific American, 193, 33-35.

Bicchieri, C. (2006). The Grammar Of Society. New York, NY: Cambridge University Press.

Bicchieri, C. (2008). "The fragility of fairness: An experimental investigation on the conditional status of pro-social norms.” Philosophical Issues, 18, 229-248.

Bordia, P. (1997). "Face-to-Face Versus Computer-Mediated Communication: A Synthesis of the Experimental Literature.” The Journal of Business Communication, 34, 99-120.

Burger, J.M., Soroka, S., Gonzago, K., Murphy, E. and Somervell, E. (2001). “The effect of fleeting attraction on compliance to requests." Personality and Social Psychology Bulletin, 27, 1578-1586.

Cialdini, R.B., and Goldstein, N. J. (2004). Social influence: Compliance and conformity. Annual Review of Psychology, 55, 591-621. 
Cialdini, R., Kallgren, C. and Reno, R. (1991). “A focus theory of normative conduct: A theoretical refinement and reevaluation of the role of norms in human behavior." Advances in Experimental Social Psychology, 24, 201-234.

Cinnirella, M., and Green, B. (2007). “Does 'cyber-conformity' vary cross-culturally? Exploring the effect of culture and communication medium on social conformity." Computers in Human Behavior, 23, 2011-2025.

Elster, J. (1990). “Norms of Revenge.” Ethics, 100, 826-885.

Elster, J. (2009). "Social norms and the explanation of behavior.” In P. Hedström and P. Bearman, eds., The Oxford Handbook of Analytical Sociology (pp. 195-217). Oxford: Oxford University Press.

Greene, J.D., Nystrom, L.E., Engell, A.D., Darley, J.M., and Cohen, J.D. (2004). "The neural bases of cognitive conflict and control in moral judgment." Neuron, 44, 389-400.

Greene, J.D., Sommerville, R., Nystrom, L., Darley, J., and Cohen, J. (2001). "An fMRI Investigation of Emotional Engagement in Moral Judgment.” Science, 293, 2105-2108.

Haidt, J. (2001). “The Emotional Dog and Its Rational Tail: A Social Intuitionist Approach to Moral Judgment." Psychological Review, 108, 814-834.

Haidt, J., Koller, S. and Dias, M. (1993). "Affect, culture, and morality, or is it wrong to eat your dog?” Journal of Personality and Social Psychology, 65, 613-628.

Henrich, J., and Boyd, R. (1998). The evolution of conformist transmission and the emergence of between-group differences. Evolution \& Human Behavior, 19, 215-241.

Hiltz, S.R., Johnson, K. and Turoff, M. (1986). "Experiments in group decision making communication process and outcome in face-to-face versus computerized conferences." Human Communication Research, 13, 225-252.

Hogg, M.A. and Reid, S.A. (2006). "Social identity, self-categorization, and the communication of group norms." Communication Theory, 16, 7-30.

Huebner, B., Dwyer, S. and Hauser M. (2009). "The role of emotion in moral psychology." Trends in Cognitive Science, 13, 1-6. 
Kelly, D. (2011) Yuck! The Nature and Moral Significance of Disgust. Cambridge, MA: The MIT Press.

Kelly, D., Stich, S., Haley, K., Eng, S. and Fessler, D. (2007). "Harm, affect and the moral/conventional distinction." Mind and Language, 22, 117-131.

Knobe, J. (2003). "Intentional Action and Side Effects in Ordinary Language." Analysis, 63, 190193.

Van Lankveld, G., Spronck, P., van den Herik, J. and Arntz, A. (2011). "Games as Personality Profiling Tools.” In M. Preuss (Ed.), Proceedings of the 2011 IEEE Conference on Computational Intelligence in Games (CIG'11), (pp. 197-202).

Laporte, L., van Nimwegen, C. and Uyttendaele, A.J. (2010). "Do people say what they think: social conformity behavior in varying degrees of online social presence.” In E.B. Hvannberg, M.K. Lárusdóttir, A. Blandford and J. Gulliksen, eds., Proceedings of NordiCHI 2010, (pp. 305-314).

Milgram, S. (1963). "Behavioral study of obedience." Journal of Abnormal and Social Psychology, 67, 371-378.

Nado, J., Kelly, D. and Stich, S. (2009). "Moral Judgment.” In J. Symons and P. Calvo, eds., The Routledge Companion to the Philosophy of Psychology (pp. 621-633). New York: Routledge.

Nichols, S. (2002). "Norms with feeling: Towards a psychological account of moral judgment." Cognition, 84, 221-236.

Nichols, S. (2004). Sentimental Rules: On the Natural Foundations of Moral Judgment. New York, NY: Oxford University Press.

Nucci, L. (2001). Education in the Moral Domain. Cambridge: Cambridge University Press.

Prinz, J. (2006). “The Emotional Basis of Moral Judgments.” Philosophical Explorations, 9, 29-43.

Puntoni, S., Langhe, B. de and Osselaer, S. Van (2008). "Bilingualism and the emotional intensity of advertising language.” Journal of Consumer Research, 35, 1012-1025.

Royzman, E., Leeman R., and Baron J. (2009). "Unsentimental ethics: Towards a content-specific account of the moral-conventional distinction." Cognition, 112, 159-174. 
Schnall, S., Haidt, J., Clore, G. L. and Jordan, A. H. (2008). "Disgust as embodied moral judgment.” Personality and Social Psychology Bulletin, 34, 1096-1109.

Schultz, P. W., Nolan, J. M., Cialdini, R. B., Goldstein, N. J., and Griskevicius, V. (2007). The constructive, destructive, and reconstructive power of social norms. Psychological Science, 18, $429-434$

Short, J., Williams, E. and Christie, B. (1976). The social psychology of telecommunications. London: Wiley.

Singer, P. (1972). "Famine, Affluence, and Morality." Philosophy and Public Affairs, 1, 229-243.

Smetana, J. (1993). “Understanding of social rules.” In M. Bennett, ed., The Development of Social

Cognition: The Child as Psychologist, New York : Guilford Press.

Smilowitz, M., Compton, C. and Flint, L. (1988). "The effect of computer mediated communication on an individual's judgement: A study based on the methods of Asch's social influence experiment." Computers in Human Behavior, 4, 311-321.

Sousa, P. (2009). “On testing the 'moral law”” Mind \& Language, 24, 209-234.

Sousa, P., Holbrook, C. and Piazza, J. (2009). “The morality of harm.” Cognition, 113, 80-92.

Tajfel, H., Billig, M., Bundy, R. and Flament, C. (1971). "Social Categorization and Intergroup Behaviour.” European Journal of Social Psychology, 1, 149-178.

Turiel, E. (1977). "Distinct conceptual and developmental domains: Social convention and morality.” In H. Howe and C. Keasey Lincoln, eds., Nebraska Symposium on Motivation, 1977: Social Cognitive Development, 25 (pp. 77-116). University of Nebraska Press.

Turiel, E. (1983). The development of social knowledge: morality and convention. Cambridge: Cambridge University Press.

Turiel, E. (2002). The Culture of Morality: Social Development, Context and Conflict. Cambridge: Cambridge University Press.

Wheatley, T. and Haidt, J. (2005). "Hypnotically Induced Disgust Makes Moral Judgments More Severe." Psychological Science, 16, 780-84. 
Whiten, A., Horner, V., and de Waal, F. B. (2005). "Conformity to cultural norms of tool use in chimpanzees." Nature, 437, 737-740. 


\section{Table 1}

Summary of the mean participants' judgments in the two survey conditions per item, measured on a 7-point disagree/agree- scale $(N=64)$.

\begin{tabular}{|l|c|c|c|c|c|c|c|}
\hline \multicolumn{1}{|c|}{ Item } & Moral & $\boldsymbol{S D}$ & Social & $\boldsymbol{S D}$ & Decency & $\boldsymbol{S D}$ & $\boldsymbol{\eta}_{\mathbf{p}}{ }^{2}$ \\
\hline Badness & 5.3 & 0.6 & 4.5 & 0.72 & 5.0 & 1.1 & $.30^{*}$ \\
\hline Disgust & 4.9 & 0.73 & 3.7 & 0.96 & 5.9 & 0.79 & $.84^{*}$ \\
\hline Time/Place & 2.9 & 0.72 & 3.7 & 0.86 & 3.3 & 1.1 & $.28^{*}$ \\
\hline Authority & 2.9 & 0.61 & 3.5 & 0.81 & 2.9 & 1 & $.21^{*}$ \\
\hline
\end{tabular}

Note. ${ }^{*} p<.05$ 


\section{Table 2}

Examples of experimental scenarios involving a violation of normative behavior

\begin{tabular}{|l|l|}
\hline Norm Type & Scenario \\
\hline Moral & $\begin{array}{l}\text { One day Lauren invites Aaron to her place for tea. } \\
\text { Aaron accepts even though he doesn't know } \\
\text { Lauren very well. They are having their tea when } \\
\text { Lauren has a sexual urge. She wants to have sex } \\
\text { with Aaron. Aaron is not willing, he tells Lauren, } \\
\text { tries to fend her off, but he can't. Lauren tears off } \\
\text { Aaron's clothes and she has with him. } \\
\text { On a scale between 1 and 7 how stronly do you } \\
\text { approve/disapprove of Lauren having sex with } \\
\text { Aaron? }\end{array}$ \\
\hline Social & $\begin{array}{l}\text { Michiru, Mauro and Robert are at the pub } \\
\text { together. Mauro buys the second. When they have } \\
\text { finished their second drink, Robert walks to the } \\
\text { bar and buys a drink only for himself. Michiry } \\
\text { and Mauro buy their drink only for himself. } \\
\text { On a scale between 1 and 7 how strongly do you } \\
\text { approve/disapprove of Robert buying a drink only } \\
\text { for himself? }\end{array}$ \\
\hline Decency & $\begin{array}{l}\text { Susan usually has cereals for breakfast. One } \\
\text { morning she realizes she finished her favorite } \\
\text { cereals. She has only an old pack with grubs and } \\
\text { insects inside. She puts them in a bowl and } \\
\text { microwaves it first to kill the germs. Then she } \\
\text { eats them: } \\
\text { On a scale between 1 and 7 strongly do you } \\
\text { approve/disapprove of Susan eating cereals with } \\
\text { insects and grubs for breakfast? }\end{array}$ \\
\hline
\end{tabular}




\section{Table 3}

Violations involved in the scenarios classified according to the Type of Norm

\begin{tabular}{|c|c|c|}
\hline Moral & $\begin{array}{l}1 . \\
2 . \\
3 . \\
4 . \\
5 . \\
6 . \\
7 . \\
8 . \\
9 . \\
10 .\end{array}$ & $\begin{array}{l}\text { Getting drunk while being the designated driver } \\
\text { Wife cheating on her loving husband } \\
\text { Not paying taxes in Italy } \\
\text { Catching frogs and pouring boiling oil on them } \\
\text { Woman forcing a man to have casual intercourse } \\
\text { Harming the environment to increase profits (Knobe, 2003a) } \\
\text { Buying a luxury car during famine in Ethiopia (Singer 1972) } \\
\text { Not voting in EU elections with a low turnout } \\
\text { Keeping slaves } 200 \text { years ago (Kelly et al 2007) } \\
\text { Downloading music from the Internet illegally }\end{array}$ \\
\hline Social & $\begin{array}{l}1 . \\
2 . \\
3 . \\
4 . \\
5 . \\
6 . \\
7 . \\
8 . \\
9 . \\
10 . \\
.\end{array}$ & $\begin{array}{l}\text { Having a sexual intercourse in a mosque } \\
\text { Not taking a vengeance for one's sister on Corsica (Elster 1990) } \\
\text { Coming to a dinner without a gift for the hosts } \\
\text { Enjoying rounds of drinks but not contributing (Elster 2009) } \\
\text { Not leaving a tip in a restaurant in the U.S. (Elster 2009) } \\
\text { Playing cards in a church during a funeral } \\
\text { Not sharing gained money during a game (Bicchieri 2006) } \\
\text { Making a phone call in a cinema } \\
\text { Playing further after an opponent injured in a game } \\
\text { Leaving a shopping cart in the line to shop further (Elster 2009) }\end{array}$ \\
\hline Decency & $\begin{array}{l}1 . \\
2 . \\
3 . \\
4 . \\
5 . \\
6 . \\
7 . \\
8 . \\
9 . \\
10 .\end{array}$ & $\begin{array}{l}\text { Eating parts of the deceased relatives' bodies (Kelly et al 2007) } \\
\text { Wearing a sweater that once belonged to Hitler (Rozin 2004) } \\
\text { Brother and sister making love (Haidt 2001) } \\
\text { Eating one's dog after it was killed by a car (Haidt 1993) } \\
\text { Eating cereals with insects for breakfast (Kelly 2011) } \\
\text { Sexual partners urinating on each other (Kelly 2011) } \\
\text { Bathing in chicken blood (Haidt 1993) } \\
\text { Sheep ranchers having sex with sheep (Haidt 1993) } \\
\text { Growing worms in the bedroom and eating them (Kelly 2011) } \\
\text { Spitting in glasses before drinking (Nichols 2002) }\end{array}$ \\
\hline
\end{tabular}




\section{Table 4}

Mann-Whitney tests for the conditions with high social presence $(N=33)$ and with low social presence $(N=35)$ compared to the Control condition $(N=29)$. The condition with low social presence differed from the Control condition in the case of Moral and Decency Conformity, but not for Social Conformity and the Total Conformity.

\begin{tabular}{|l|c|c|c|c|c|c|}
\hline & \multicolumn{3}{|c|}{ High SP } & \multicolumn{3}{c|}{ Low SP } \\
\hline Conformity & $U$ & $p$ & $r$ & $U$ & $p$ & $r$ \\
\hline Moral & 297.000 & .01 & -.328 & 397.000 & .13 & -.188 \\
\hline Social & 88.000 & .00 & -.701 & 297.500 & .00 & -.356 \\
\hline Decency & 184.000 & .00 & -.529 & 464.000 & .56 & -.074 \\
\hline Total & 116.000 & .00 & -.650 & 323.500 & .01 & -.311 \\
\hline
\end{tabular}

Note. $d f=2, S P=$ Social Presence

\section{Table 5}

Median Conformity differences in the three experimental conditions (low social presence, high social presence and control) by Norm Type $(N=97)$. The scores express the change in distance to the confederates' opinion, higher score indicating higher conformity $(0=$ no change).

\section{Condition}

High SP Low SP Control

$.40 \quad .20$

.60

.55

.52

.00

.00

.10

.00

$.16 \quad .00$

Statistics

Total

Note. $d f=2, S P=$ Social Presence 


\section{Table 6}

Results of the mixed ANOVA with the three experimental conditions and three norm types as independent variables and consistency of answers as the dependent variable (a higher value indicates less consistent answers), Norm Type: $F(2,188)=9.95, p<.001$, partial eta squared $=$. 10; Condition: $F(2,94)=6.24, p=.003$, partial eta squared $=.12$; Norm Type * Condition n.s. $A$ pairwise comparison analysis showed a significant difference between moral and social, and moral and decency norms, but no difference between social and decency norms.

Condition

\begin{tabular}{|c|c|c|c|c|c|c|c|}
\hline \multirow[b]{2}{*}{ Norm Type } & \multirow[t]{2}{*}{$\mathrm{N}$} & \multicolumn{2}{|c|}{ With SP } & \multicolumn{2}{|c|}{ Without SP } & \multicolumn{2}{|c|}{ Control } \\
\hline & & Mean & SD & Mean & SD & Mean & $\overline{\mathrm{SD}}$ \\
\hline Moral & 10 & 0.81 & 0.38 & 0.78 & 0.32 & 0.63 & 0.34 \\
\hline Social & 10 & 1.08 & 0.41 & 0.92 & 0.43 & 0.73 & 0.31 \\
\hline Decency & 10 & 1.06 & 0.50 & 0.82 & 0.31 & 0.76 & 0.43 \\
\hline
\end{tabular}

Note. $\mathrm{SP}=$ Social Presence 